\title{
IDENTIFICANDO PROBLEMAS DE ROTATIVIDADE DE PESSOAL ATRAVÉS DE FERRAMENTAS DA QUALIDADE
}

\author{
Marcela Avelina Bataghin Costa', Mariana Gianei² \\ ${ }^{1}$ Instituto Federal de Educação, Ciência e Tecnologia - IFSP, curso Superior de Tecnologia em Processos Gerenciais, \\ Campus de São Carlos, SP. ${ }^{2}$ Universidade Federal de São Carlos, Departamento de Pedagogia, São Carlos, SP. E- \\ mail:marigianei@hotmail.com; marcela.bataghin@ifsp.edu.br
}

\section{RESUMO}

O departamento de gestão de pessoas é responsável por administrar, promover e reter os talentos na empresa. $O$ presente trabalho tem como objetivo principal verificar a relação entre a rotatividade e a gestão de pessoas em uma determinada empresa. Para tanto realizou-se uma revisão bibliográfica seguida de estudo de caso. Buscando alcançar o objetivo foram utilizadas Ferramentas da Qualidade com o intuito de identificar a fonte de problemas administrativos que levavam a rotatividade dos funcionários. A primeira ferramenta utilizada foi o brainstorming que auxiliou na identificação e classificação dos problemas e suas causas. Em seguida foi utilizado o Diagrama de Causa e Efeito com adição de cartões -CEDAC para propor melhorias a partir da classificação gerada.

Palavras-chave: Gestão de pessoas. Rotatividade. Ferramentas da qualidade. CEDAC, Brainstorming.

\section{IDENTIFYING AND PROBLEMS OF TORNOVER OF PEOPLE THROUGH QUALITY TOOLS}

\section{ABSTRACT}

The People Management departament is responsible for managing, promoting and retaining talent in the company. The present paer has as main objective the relation between the rotation and the management of people in a company. To do so, a complete case study bibliography is presented. Seeking immediate to the fixed tools Frequently the quality of the specific resource of the office that have led to the turnover of employees. The first tool used was the brainstorming that aided in the identification and classification of problems and their causes. Then, the Cause Diagram and the Effect of CEDAC Tables were used to provide the generated classification.

Keywords: Management people. Turnover. Quality tools. CEDAC. Brainstorming.

\section{INTRODUÇÃO}

Encarregada por "agregar valores humanos, integrá-los e alinhá-los em suas atividades," (CHIAVENATO, 2009, p.2) a Gestão de Pessoas é uma das grandes responsáveis pelo sucesso da empresa. É considerada também uma extensão do tema Recursos Humanos.

Para, Souza, Paixão e Souza (2011 apud PINHEIRO, 2013, p. 3):

Com intensidade cada vez maior, as organizações passam a investir na gestão de pessoas, indo além dos conhecidos e tradicionais processos burocráticos de recursos humanos.

Assim, infere-se que ao investir na gestão de pessoas, a organização investe no futuro dos seus profissionais e em sua permanência no mercado, já que o setor seleciona e desenvolve talentos e ajuda a evitar diversos fenômenos negativos, como por exemplo, a rotatividade - turnover (PINHEIRO, 2013).

Segundo o Departamento Intersindical de Estatísticas e Estudos Socioeconômicos, DIEESE (2014) em 2012 cerca de $45 \%$ das taxas de rotatividade ocorreram com menos de seis meses de vigência do contrato de trabalho. Ainda sobre as taxas de rotatividade, vista sob a ótica setorial observou-se que no 
mesmo ano a atividade econômica de Serviços apresentou índice de rotatividade próximo a 39\% no Brasil. Em 2015 este índice foi de 58,7\%.

$\mathrm{O}$ alto índice de rotatividade pode ser um custo oneroso às empresas. Para Pinheiro $(2013$, p.4) a saída de colaborador, reposição e treinamento para o novo colaborador geram custos financeiros e econômicos para a empresa, além de causar a queda na produtividade e desempenho.

Com isso, o presente trabalho tem como problema de pesquisa verificar: qual a relação existente entre a rotatividade de funcionários e a Gestão de Pessoas em uma empresa do segmento educacional de idiomas, localizada no interior de São Paulo? O objeto deste estudo foi uma organização do segmento educacional de idiomas, onde previamente identificou-se um problema de rotatividade entre os professores da empresa. Deste modo seu principal objetivo é baseado no problema identificado propor a melhorias utilizando o Diagrama de Ishikawa para identificar a(s) causa(s) da rotatividade de funcionários na escola e especificamente: verificar se de fato a rotatividade de funcionários está relacionado a problemas de gestão de pessoas; fazer um brainstorming para embasar um Diagrama de Causa e Efeito e finalmente propor melhorias o CEDAC, Diagrama de Causa e Efeito com Adição de Cartões.

Para Limongi França $(2009$, p.5) a "Gestão de Pessoas é um conjunto das forças humanas voltadas para atividades produtivas, gerenciais e estratégicas dentro de um ambiente organizacional". Assim, a gestão de RH é importante para a empresa, pois analisada pelos aspectos comportamentais e administrativos, relaciona-se com todos os setores de uma organização de modo a desenvolver e orientar os funcionários, além de buscar maximizar o potencial de trabalho (RIBEIRO, 2005).

Como a Gestão de Pessoas busca a interação e associação do funcionário com a empresa, o setor deve busca compreender as diversidades existentes na organização. As pessoas são diferentes entre si, se comportam baseadas no que percebem, vivem, trabalham, além de serem influenciadas por outras pessoas (LIMONGI FRANÇA, 2009). Para tanto é necessário que a área de RH da empresa estude a comunidade, a cultura local e procure entender as necessidades de seus funcionários para poder criar comprometimento e fidelidade com a empresa, justificando portanto, esta pesquisa

\subsection{Método de pesquisa}

Esta pesquisa caracteriza-se por ser de natureza aplicada já que é voltada à aquisição de conhecimento com vistas à aplicação numa situação específica (GIL, 2010, p. 27). Além disso, o presente trabalho caracteriza-se por ser uma revisão bibliográfica seguida de estudo (GIL, 2010). É descritiva, pois "tem a finalidade de identificar possíveis relações entre as variáveis" (GIL, 2010). Utilizando a classificação de Prodanov e Freitas (2013, p.70) a pesquisa possui uma abordagem qualitativa, isto é, predomina a interpretação dos fenômenos e dados adquiridos, atribuindo significados à pesquisa. Utiliza amostra intencional sendo esta um tipo de abordagem não probabilística (PRODANOV; FREITAS 2013, p. 99). Para coleta de dados, inicialmente foi realizado um brainstorming de modo a levantar as causas relevantes que originam o problema de rotatividade. Após o uso desta ferramenta foi aplicado o Diagrama de Causa e Efeito, para poder identificar de forma clara os problemas que influenciam o problema principal rotatividade- e assim poder analisá-los de forma mais clara e direta e poder propor um solução ao(s) problema(s) encontrado(s).

\section{EVOLUÇÃO DE RECURSOS HUMANOS}

\subsection{Recursos Humanos}

Administração de Recursos Humanos é o departamento responsável por gerenciar as relações dos funcionários com a organização. Albuquerque e Leite $(2009$, p.15) atribuem que:

Administração de RH é construir e reter talentos na organização, produzindo e dando o máximo de si, de modo positivo e favorável aos objetivos da organização, em consonância com os objetivos individuais, alcançando eficiência, eficácia e efetividade.

Para esta finalidade, é necessário que o departamento de Recursos Humanos englobe diversos setores da organização para poder desenvolver uma estratégia adequada que permita, assim, atrair, reter e desenvolver pessoas (RIBEIRO, 2005, p.3).

A Gestão de Pessoas busca focar na representatividade dos funcionários e dos parceiros da organização, onde todos são partes essenciais para o produto final. Por ser uma prática situacional, ou seja, 
dependente da cultura local, processos internos, tecnologia, contexto ambiental, varia de acordo com estrutura organizacional adotada. De acordo com Chiavenato (2009, p.2):

[...] Administração de Recursos Humanos constitui uma área extremamente sensível a três aspectos organizacionais: a estrutura organizacional escolhida, a cultura organizacional dominante e o estilo de gestão adotado. Por isso, ela é contingencial e situacional.

Frente à competitividade de mercado, globalização e as crescentes mudanças industriais, tornou-se necessário um bom profissional responsável por desenvolver a área de Recursos Humanos.

Assim, observa-se que empresas que possuem excelência e liderança no mercado têm um departamento de Recursos Humanos desenvolvido e dinâmico. Baseada nessa ideia, Pinheiro (2013) argumenta que o conhecimento é um diferencial exigido pelas organizações e que as empresas têm a necessidade de investir em seus funcionários acrescentando habilidades, competências e comportamento já que todo o recurso humano envolvido - cliente, funcionários e fornecedores- são essenciais para o sucesso da organização.

Em uma época que a competitividade de mercado, globalização e a tecnologia estão modificando o mercado e promovendo novos desafios o setor de Gestão de Pessoas precisa administrar dificuldades relacionadas às pessoas, evitando problemas como rotatividade e que podem prejudicar o desempenho de uma empresa (RIBEIRO, 2005).

\subsection{Rotatividade}

Também conhecido como Turnover, refere-se ao fluxo de entradas e saídas de pessoas em uma organização de forma voluntária ou involuntária, em um determinado período de tempo, influenciando significativamente os processos e resultados das empresas por ser um aspecto muito importante na dinâmica organizacional (PINHEIRO, 2013, p.4). Segundo Bohlander e Snell (2010) o desligamento de um empregado desqualificado pode ser benéfico para a empresa, já que a empresa teria o custo de treinamento e capacitação do individuo. Porém segundo os mesmos autores, a substituição de um empregado é um processo demorado e oneroso gerando altos custos á empresa.

Dentre as principais causas da rotatividade, Marques (2016) destaca as seguintes:

Descontentamento com a política e metas da empresa; falta de motivação nos casos em que a empresa não oferece benefícios ou incentivos que mostrem a valorização dos funcionários; falta de plano de carreira, fazendo com a que as pessoas se desmotivem na medida em que percebem que não terão oportunidades de crescimento; baixa remuneração, principalmente quando os funcionários não recebem remuneração justa a sua qualificação, e a falta de investimentos e benefícios que acabam influenciando na evasão de pessoas; ambiente de trabalho hostil que promove a queda na produtividade do funcionário e consequentemente sua saída do emprego assim que surge uma nova e melhor oportunidade.

\subsection{Gestão de Recursos Humanos e Qualidade}

Em plena $4^{a}$ Revolução industrial, onde a automação e tecnologia são as principais tendências nas grandes potências, antigos problemas precisam ser solucionados. Assim as empresas e seus setores administrativos devem buscar a melhoria contínua dos seus processos, serviços e pessoal. A Gestão de Pessoas procura atentar às questões relacionadas aos funcionários na organização, voltada às atividades estratégicas, produtivas e gerenciais da empresa (LIMONGI FRANÇA, 2009, p.5). As pessoas são de modo geral diferentes e resolver problemas relacionados a elas não é simples, já que cada um possui um comportamento próprio e tem reações diferentes a cada estimulo. Para isso, desenvolveram-se ferramentas que orientam os profissionais a uma melhoria de forma geral, tanto no desenvolvimento do pessoal como também no processo produtivo. Possuindo tal finalidade de "permitir ao usuário encontrar uma solução ao problema proposto" (PALADINI, 2012, p. 353), as Ferramentas da Qualidade embora pensada para a área de qualidade hoje tem sua utilidade nas mais diversas áreas de uma organização.

\section{FERRAMENTAS DE QUALIDADE E SUA APLICAÇÃO EM GESTÃO}

Compreender os fundamentos e significados de Gestão da Qualidade são insuficientes para garantir o êxito na aplicação, para tanto, houve a necessidade de desenvolver-se ferramentas e estratégias que viabilizassem a efetiva aplicação da Gestão de Qualidade permitindo a obtenção de resultados imediatos e notáveis (PALADINI, 2012, p. 352). De modo conceitual, ferramentas da Gestão da Qualidade: São 
mecanismos simples para selecionar, implantar ou avaliar alterações no processo produtivo por meio de análise objetivas de partes bem definidas deste processo. Evidentemente, o objetivo das alterações é gerar melhorias (PALADINI, 2012, p.352).

De forma mais ampla, "ferramentas são métodos estruturados de modo consistente para viabilizar a definição de melhorias que possam vir a ser implantadas em partes definidas do processo produtivo" (PALADINI, 2012, p. 354), atuando na fase anterior e posterior da implantação do processo. Apesar de existirem diversas ferramentas, em diferentes graus, elas apresentam características semelhantes (PALADINI, 2012): facilidade de uso; lógica de operação; sequência correta de ações; alcance visual; etapas para implementação e foco na solução. Dentre os mecanismos existentes, a presente pesquisa utilizou-se das seguintes ferramentas: Brainstorming e Diagrama de Causa e Efeito com adição de Cartões (CEDAC).

\subsection{Brainstorming}

Segundo Santo (2015, p.1) Brainstorming (tempestade de ideias) é uma técnica usada para gerar ideias rapidamente em quantidade e pode ser empregada em várias situações. A ferramenta auxilia na geração de ideias e na busca de soluções aos problemas. Antes de se iniciar o processo, é necessário que o grupo de participantes eleja um líder, alguém com condições e conhecimentos para conduzir a reunião, monitorando o ritmo da discussão ajudando a equipe a manter o foco. A partir de então são geradas as possíveis causas para a os problemas em questão. Nesta técnica, as ideias devem ser geradas rapidamente, sem interferências, retaliações ou punições. Todas as ideias são anotadas e as mais coerentes selecionadas ao final das seções (COSTA, 2014).

\subsection{Diagrama de Causa e Efeito}

Também conhecido como gráfico de Espinha de Peixe ou Diagrama de Ishikawa, foi criado pelo engenheiro japonês Kaoru Ishikawa (1915-1989) no ano de 1943. O gráfico permite ao usuário entender como um processo produtivo opera, separando as causas dos efeitos, além de criar um paradigma entre eles (OAKLAND, 1994, p. 227). A estrutura do diagrama é semelhante a uma espinha de peixe, onde o eixo principal mostra o principal problema e as espinhas às principais ações e causas para que isso ocorra, sendo que as sequências das ações conduzem ao resultado final de cada uma das causas, permitindo a visualização da relação entre as causas e os efeitos delas decorrentes (PALADINI, 2012).

Segundo Oakland (1994), o efeito ou problema que está sendo investigado aparece na ponta de uma flecha horizontal, como mostra a Figura 1.

Figura 1. Diagrama de Causa e Efeito

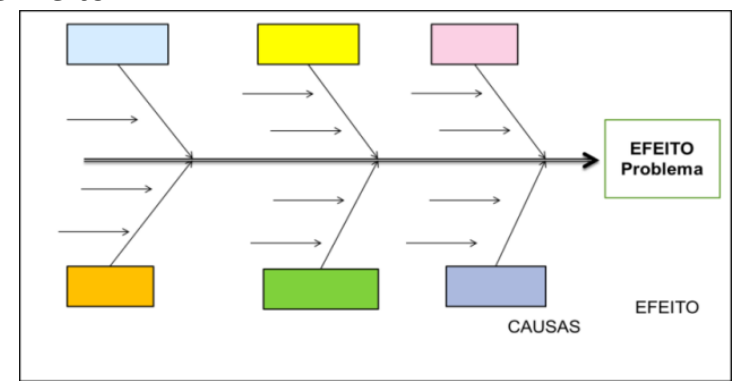

Fonte: Adaptado de Oakland (1994)

Como a "ideia de Ishikawa era fazer com que as pessoas pensassem sobre as causas e razões possíveis que fazem com que um problema ocorra" (COSTA e TOLEDO, 2016), ela pode ser aplicada para a solução de problemas de diversas áreas. Para dar inicio ao processo, o grupo de funcionários afetados pelo problema, liderados por alguém, reúnem-se para realizar um. De forma geral, o Ishikawa pode seguir o seguinte roteiro (PALADINI, 2012):

Identificar o problema a ser eliminado; colocar o problema do lado direito do diagrama, no eixo central; o grupo começa a sugerir e determinar as causas deste problema; diferenciar essas causas que contribuem ao problema colocando-as nas espinhas do diagrama; classificar em causas principais e causas básicas (influenciam as principais); verifica-se a veracidade das causas, passando por uma revisão crítica para avaliar se é compatível com a realidade da empresa; em seguida, é a fase de experimentação, na qual cada causa vai sendo testada e analisada com mais detalhes, o diagrama vai sendo refinado; classificando 
qual a relevância e incidência do problema é possível buscar soluções para resolvê-lo, podendo definir então, as causas do problema.

\subsubsection{Diagrama de Causa e Efeito com adição de Cartões}

Uma variação do Diagrama de Causa e Efeito, desenvolvida na empresa japonesa Sumitomo Electric, é o Diagrama de Causa e Efeito com Adição de Cartões - CEDAC (Cause and Effect Diagram with Addiction of Cards).

De acordo com Oakland (1994, p. 228), o diagrama é muito semelhante ao original. Um líder do projeto é selecionado e estabelece o alvo da melhoria, utilizando- se de cartões de cores diferentes- para melhor visualização e compreensão- onde serão escritos as causas e as ideias. As causas são reunidas e colocadas à esquerda do diagrama, enquanto os cartões com ideias de melhoramento são formulados e colocados à direita das espinhas das causas, conforme Figura 2.

Figura 2. Diagrama de Causa e Efeito com Adição de Cartões

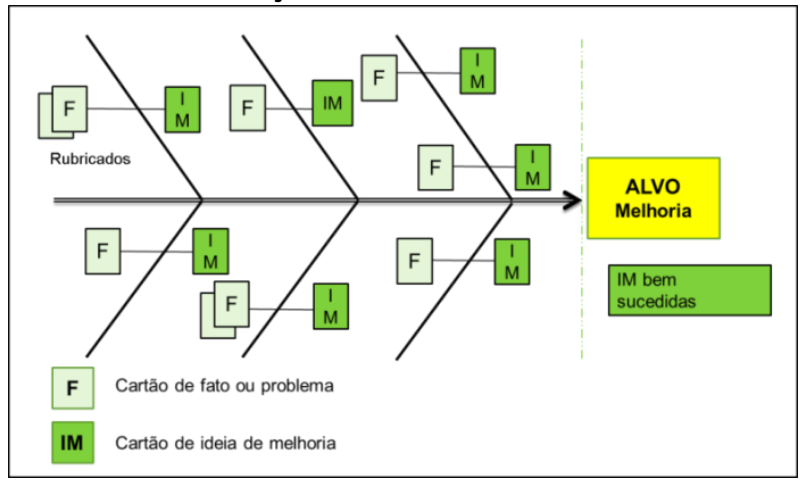

Fonte: Adaptado de Oakland (1994)

As ideias são selecionadas e avaliadas quanto ao seu conteúdo e praticabilidade (OAKLAND, 1994, p.229) e as melhores se tornam parte do processo de melhoria da empresa.

\section{ESTUDO DE CASO}

\subsection{Caracterização da Unidade estudada}

A unidade objeto de estudo localiza-se no interior do estado de São Paulo. De acordo com as informações coletadas teve as suas atividades iniciados em fevereiro de 2014. Por ser uma franquia, a metodologia e material utilizado segue um padrão estabelecido e, apesar de oferecer seis cursos de línguas, - com duração em média de dois anos- atualmente, predomina a procura pelo curso de Inglês.

Atualmente a empresa conta com um total de sete funcionários: o mantenedor da unidade, quatro professores e duas secretarias. De todos os funcionários, apenas as secretárias residem na cidade. Somente uma delas é contratada formalmente pela empresa. Os demais residem na cidade de São Carlos. Atualmente, a empresa não possui um contrato formal com os professores, apenas acordos informais de comprometimento com a escola.

Durante seus 3 anos de existência na cidade, aumentou a demanda pelos serviços oferecidos o que permitiu que a empresa expandisse seus negócios, incluindo a mudança para um novo prédio, além de aumentar o seu quadro funcionários, como verificado no Gráfico 1.

A rotatividade de professores aumentou ao longo dos anos. Em 2014, um (1) docente foi demitido, em 2015 foram dois (2) demitidos e em 2016, um total de 3 professores foram demitidos. O Gráfico 2 mostra as possíveis causas dessas demissões. 
Gráfico 1. Número de funcionários contratados por ano

Fonte: Os autores (2017)

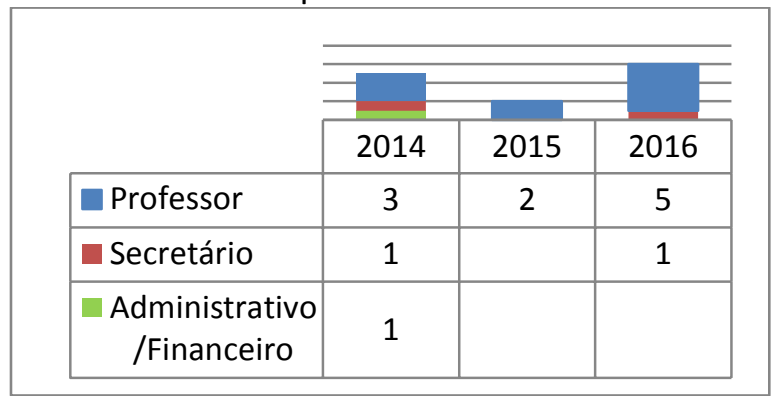

Gráfico 2. Possíveis motivos para as demissões

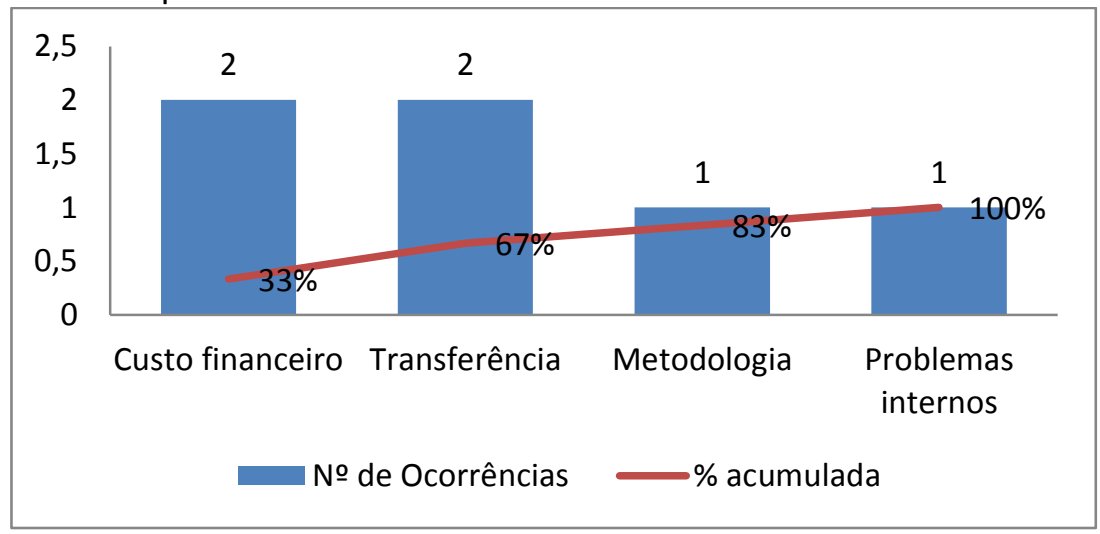

Fonte: Os autores (2017).

\subsubsection{Análise das informações qualitativas}

Mediante os dados fornecidos pela unidade, apurou-se o histórico sobre os possíveis motivos que levaram as demissões. Em primeiro, destacam-se os custos financeiros para os professores, já que o salário oferecido pelos serviços não compensava financeiramente o tempo e as viagens, muitas vezes os professores acabam pagando para trabalhar. Logo após, é possível observar a transferência de professores a outras unidades, resultante das vantagens que outras unidades ofereciam em relação a esta. Em seguida, tem se a demissão pela não adaptação à metodologia de ensino usada pela empresa e por fim, um problema interno que culminou da demissão de um professor.

Com a caracterização da empresa, constatou-se que não há um setor ou funcionário responsável pela área de Gestão de Pessoas, podendo ser um fator que contribui para a rotatividade. Com essa postura, possivelmente, um profissional contratado especificamente para desenvolver a Gestão de RH na unidade, conseguiria vir a auxiliar na tomada de decisões e soluções de problemas, de modo a ajudar ou reduzir o problema de rotatividade.

\subsection{Aplicação do Brainstorming}

Baseado nas informações adquiridas na empresa sobre os possíveis motivos das demissões anteriores realizou-se um brainstorming a fim de identificar as causas que levaram ao aumento da rotatividade. Baseado nas etapas propostas pelo site Empreendedor Universitário (2013), as etapas seguidas para realização do brainstorming foram: identificação do problema de rotatividade entre os professores na empresa; reunião com os envolvidos, no caso com os professores, para discussão e aplicação do brainstorming; explicar as regras aos participantes, no caso, explanar os critérios da pesquisa aos professores; geração de ideias entre os envolvidos no processo, estimulados por um roteiro préformulado; após a execução da ferramenta, fez-se a seleção e agrupamento das ideias adquiridas.

\section{RESULTADOS DO BRAINSTORMING E PROPOSTAS DE MELHORIA}

\subsection{Aplicação do Diagrama de Causa e Efeito}

Após organizar as informações obtidas com o brainstorming foi possível chegar aos principais problemas que afetam aos professores. Sendo assim foi possível coloca-los no Diagrama de Causa e Efeito 
para melhor análise. Foram adotadas as quatro categorias de classificação conhecido como os 4P's: Políticas, Procedimentos, Pessoal e Planta (layout), pois permitem uma analise mais dinâmica das informações, Figura 3.

Figura 3. Aplicação do Diagrama de Causa e Efeito

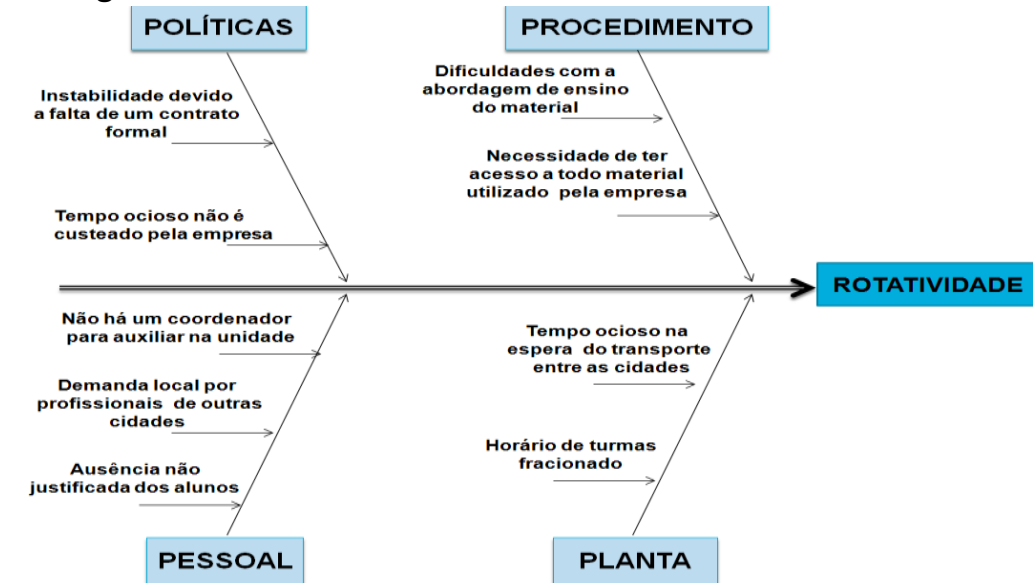

Fonte: Os autores (2017).

\subsubsection{Aplicação do Diagrama de Causa e Efeito com adição de Cartões}

Depois de organizar e classificar as informações no Diagrama de Causa e Efeito aplicou-se o Diagrama de Causa e Efeito com Adições de Cartões (CEDAC), Figura 4, com o intuito de propor uma solução a cada problema encontrado durante o brainstorming.

Assim, para os problemas encontrados foram propostas as seguintes melhorias:

A. Planejamento dos tributos da empresa. Contratar um contador/consultor que ajude a verificar em qual classificação de Pequeno Negócio ele se encaixa melhor. Utilizando o sistema MEI, a empresa está restrita a apenas um empregado contratado, com isso, se for viável a mudança para a opção de Microempresa será possível contratar mais funcionários.

B. O tempo em que o professor fica na escola deve ser custeado pela empresa;

C. Contratar um funcionário que realize a Gestão de Pessoas dentro da empresa;

Figura 4. Aplicação do Diagrama de Causa e Efeito com Adição de Cartões

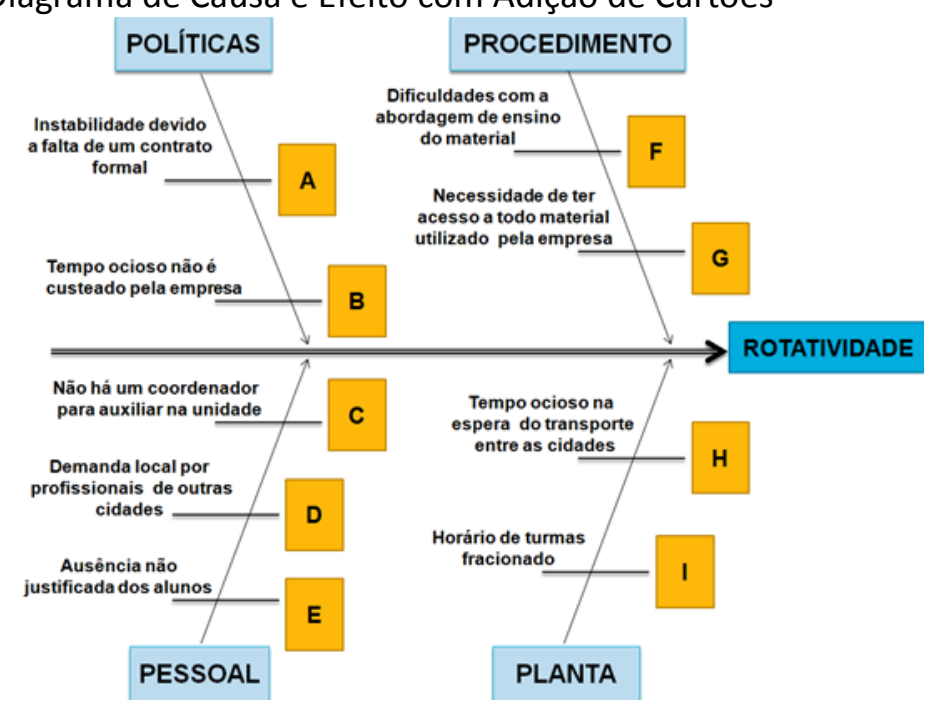

Fonte: Os autores (2017).

D. Este é um fator externo, que não está sob o controle da empresa. Contudo, há sugestão de investir no marketing da empresa ressaltando as competências e qualificações dos professores locais, demonstrando a seu público a confiabilidade da empresa e responsabilidade dos professores;

E. $\quad$ Cobrar maior responsabilidade do aluno em relação às presenças; 
F. Treinamento e acompanhamento pedagógico com os professores para auxiliar no entendimento do material;

G. Para cada idade existe um material correspondente, porém em alguns casos, a capacidade de aprendizagem do aluno não corresponde às lições propostas - tanto por dificuldade como facilidade de entendimento da matéria. Assim, o acesso a todo o material disponível permite uma melhor avaliação do conteúdo, em que o professor com a ajuda de um profissional - um caso em que se faz necessário o amparo de um coordenador pedagógico e um psicólogo- possam analisar o perfil do estudante e verificar qual o material mais apropriado;

H. Planejar as aulas de modo que os horários do trabalho dos professores fiquem mais próximos aos horários do transporte para evitar o tempo ocioso.

I. Planejar melhor as aulas, buscando ajustar os horários á disponibilidade dos alunos;

J. Necessidades dos professores e não apenas dos alunos, procurando marcar horários de aulas mais próximos entre si.

\section{CONSIDERAÇÕES FINAIS}

Os princípios da Gestão de Pessoas consistem em gerir de forma adequada o capital humano da empresa do, recrutamento á retenção, além, de responsabilizar-se por solucionar problemas administrativos, como a rotatividade (RIBEIRO, 2005). Atuando na área do ensino de idiomas, a empresa estudada tem tido aumento da demanda pelos serviços oferecidos, contudo, averiguou-se um crescente índice de rotatividade entre seus professores.

Observou-se que não havia um funcionário responsável por executar especificamente as funções de um gestor de RH na unidade, desse modo, infere-se que a ausência de um gestor competente para ajudar os funcionários da empresa, incentivasse na rotatividade dos professores. Ao realizar o brainstorming identificou-se as prováveis possibilidades que influenciariam os professores a saírem da empresa, por isso, para melhor visualização e analise dos dados, as informações adquiridas foram organizadas no Diagrama CEDAC. Em seguida, foram propostas melhorias a cada uma delas com a finalidade de minimizar ou extinguir o problema principal, porém ao realizar esse processo, reforçou-se a ideia da necessidade de um funcionário que forneça um apoio complementar, pois a presença de um coordenador fez se necessária em determinados momentos para auxilio e suporte na contribuição das resoluções das causalidades.

De acordo com Ribeiro (2005) empresas que utilizam a Gestão de Pessoas como parte de sua estratégia, mesmo que recente conseguem perceber melhorias, como a diminuição da rotatividade.

\section{REFERÊNCIAS}

ALBUQUERQUE, L. G.; LEITE, N. P. Gestão estratégica de pessoas: conceito, evolução e visão. In: ALBUQUERQUE, L. G.; LEITE, N. P. Gestão de pessoas: perspectivas estratégicas. São Paulo: Atlas, 2009. p. 3-16.

BOHLANDER, G.; SNELL, S. Calculando rotatividade e absentismo. In: BOHLANDER, G.; SNELL, S. Administração de recursos humanos. São Paulo: Cengage Learning, 2010. p. 79-83.

CHIAVENATO, I. Sistema de administração de recursos humanos. In: CHIAVENATO, I. Treinamento e desenvolvimento de recursos humanos: como incrementar talentos na empresa. 7. ed. Barueri: Manole, 2009. p. 1-14.

COSTA, M. A. B. Material didático. Ferramentas de gestão da qualidade. São Carlos, 2016.

COSTA, M. A. B. Análise de práticas do pré-desenvolvimento de novos produtos: estudo de múltiplos casos. 2014. 258 p. Tese (Doutorado em Engenharia de Produção) - Universidade Federal de São Carlos, São Carlos, 2014. Disponível em https://repositorio.ufscar.br/bitstream/handle/ufscar/3453/6457.pdf?sequence=1. Acesso em: 19 ago. 2016. 
COSTA, M. A. B; TOLEDO, J. C. O pré desenvolvimento de novos produtos e sua função essencial: modelos, técnicas, indicadores de desempenho e casos. São Carlos: Rima. 2016.

DIEESE. Rotatividade e políticas públicas para o mercado de trabalho. Departamento Intersindical de Estatística Estudos Socioeconômicos. São Paulo: DIEESE, 2014. 140 p. Disponível em: http://www.dieese.org.br/livro/2014/livroRotatividade.pdf. Acesso em: 6. set. 2016.

GIL, A. C. Como elaborar projetos de pesquisa. 5 ed. São Paulo: Atlas, 2010.

LIMONGUI FRANÇA, A. C. Práticas de recursos humanos - PRH: conceitos, ferramentas e procedimentos. São Paulo: Atlas, 2009. https://doi.org/10.1590/S1415-65552008000200016

MARQUES, J. R. Rotatividade de funcionários: Entenda as causas. 2016. Disponível em: http://www.ibccoaching.com.br/portal/rh-gestao-pessoas/causas-rotatividade-de-funcionarios/. Acesso em: 11 ago. 2016.

OAKLAND, J. S. Gerenciamento da qualidade total. São Paulo: Nobel, 1994.

PALADINI, E. P. Gestão da qualidade: teoria e casos. 2 ed. Rio de Janeiro: Elsevier: 2012.

PINHEIRO, A. P. Causas e Efeitos da Rotatividade de Pessoal/Turnover: Estudo de caso de uma microempresa do setor de educação. 2013. 13 p. Disponível em: http://www.aedb.br/seget/arquivos/artigos13/58618723.pdf. Acesso em: 11 ago. 2016.

PRODANOV, C. C.; FREITAS, E. C. Metodologia do trabalho científico: métodos e técnicas da pesquisa e do trabalho acadêmico. 2 ed. Novo Hamburgo: Feevale, 2013.

RIBEIRO, A. L. Gestão de Pessoas. São Paulo: Saraiva 2005.

SANTO. R. Brainstorming: Tempestade de idéias (BS - TI) ou Como tirar seu time do "cercadinho mental". 2015. Disponível em: http://www.bibliotecas.sebrae.com.br/chronus/ARQUIVOS_CHRONUS/bds/bds. nsf/741A876FE828908203256E7C00614A23/\$File/NT00002206.pdf. Acesso em: 28 out. 2016. 